\title{
ARTIGO DE REVISÃo Neuroimagem na dependência de jogos eletrônicos: uma revisão sistemática
}

\author{
Neuroimaging in video game addiction: a systematic review \\ Igor Lins Lemos', Paula Rejane Beserra Diniz², Julio Fernando Prieto Peres ${ }^{3}$, Everton Botelho Sougey'
}

\section{Palavras-chave \\ Dependência de jogos eletrônicos, neuroimagem, jogos eletrônicos, uso de substâncias, revisão.}

\section{RESUMO}

Objetivo: Realizar revisão sistemática de manuscritos que utilizaram a neuroimagem no estudo da dependência de jogos eletrônicos, a fim de identificar as principais regiões cerebrais alteradas. Métodos: Foram realizadas buscas nos seguintes bancos de dados: ScieELO, BVS, Lilacs, Science Direct On Line e PubMed. Não houve data mínima para a pesquisa, sendo considerados os artigos encontrados até julho de 2013. Os descritores utilizados para a presente revisão sistemática da literatura foram: "PET", "SPECT", "MRI", "DTI", "EEG", "imaging", "neuroimaging", "spectroscopy", "functional magnetic ressonance", "structural magnetic ressonance", "tractography", "voxel" e "brain", individualmente cruzados com os descritores "gaming" e "video game addiction". Resultados: Dos 52 artigos encontrados, 16 foram selecionados: nove usaram fMRI, quatro usaram sRMI, um usou PET e dois usaram EEG. Em relação às alterações funcionais e estruturais, elas foram mais observadas no lobo frontal (córtex pré-frontal dorsolateral, córtex orbitofrontal, giro pré-frontal, giro frontal médio), parietal, temporal (giro para-hipocampal), núcleos da base, tálamo, ínsula e cerebelo. Conclusão: A despeito dos métodos utilizados, os estudos apontaram convergências quanto às reciprocidades cerebrais. Essas alterações neurais são semelhantes às observadas em pacientes dependentes de substâncias e de internet, especialmente durante o estado de fissura. Apesar de apenas recentemente pesquisas de neuroimagem em dependentes de jogos eletrônicos terem sido realizadas, contamos no momento com achados significativos alinhados à compreensão dos mecanismos neurais associados à dependência de jogos eletrônicos e respectiva inserção como categoria nosológica no âmbito psiquiátrico.

\section{ABSTRACT}

Objective: To perform a systematic review of manuscripts that used neuroimage to study the video game addiction in order to identify the main altered brain regions. Methods: Searches were realized in the following databases: ScieELO, BVS, Lilacs, Science Direct On Line and PubMed. There was no minimum date for the research, being considered articles founded until July 2013. The descriptors used for this systematic review were: "PET", "SPECT", "MRI", "DTI", "EEG", "imaging", "neuroimaging", "spectroscopy", "functional magnetic resonance", "structural magnetic resonance", "tractography", "voxel" and "brain", individually crossed with descriptors "gaming" and "video game addiction". Results: Of the 52 articles founded,

1 Universidade Federal de Pernambuco (UFPE), Programa de Neuropsiquiatria e Ciências do Comportamento. 2 UFPE, Departamento de Medicina Clínica, Núcleo de Telessaúde (Nutes).

3 Universidade de São Paulo (USP), Faculdade de Medicina, Instituto de Psiquiatria (IPq).

Recebido em

10/7/2013

Aprovado em

$26 / 2 / 2014$
Endereço para correspondência: Igor Lins Lemos

Rua José de Holanda, 580, ap. 603, Torre

50710-140 - Recife, PE, Brasil

E-mail: igorlemos87@hotmail.com 


\section{Keywords}

Video game addiction, neuroimaging, electronic games, substance use, review.
16 were selected: nine used fMRI, four used sRMI, one used PET and two used EEG. In relation of the functional and structural changes, they were more often observed in the frontal lobe (dorsolateral prefrontal cortex, orbitofrontal cortex, prefrontal gyrus, middle frontal gyrus), parietal, temporal (parahippocampal gyrus), basal ganglia, thalamus, insula and cerebellum.

Conclusion: Regardless the methods utilized, the studies pointed convergences regarding reciprocity brain. These neural changes are similar to those observed in patients with substance addiction and internet addiction, especially during craving. Although only recently neuroimaging researches on game addicts were realized, we count at the moment with significant findings aligned with the understanding of the neural mechanisms associated with video game addiction and its inclusion as a nosological category in psychiatric scope.

\section{INTRODUÇÃO}

A disseminação tecnológica repercutiu intensamente na dimensão individual e social, emergindo novas formas de pensar, sentir e agir na era da cibercultura. O mundo virtual passou a ser cada vez mais atrativo com a qualidade das informações e a alta resolução e miríade de cores vívidas que atraem os usuários. A utilização de som, imagem, grafismo e todas as potencialidades hipermédia cativam os jogadores, que facilmente se adaptam a estímulos irresistíveis à atenção involuntária e voluntária'.

Concomitantemente à ascensão eletrônica, é observado, na última década, o uso problemático de tecnologia, que aumentou drasticamente ${ }^{2}$, causando impactos negativos na esfera biopsicossocial dos usuários ${ }^{3}$. Dessa forma, tornou-se recorrente a queixa de isolamento social, associado ao uso problemático da internet e dos jogos eletrônicos, em consultórios psicológicos e psiquiátricos ${ }^{1-3}$.

Apesar de a dependência de jogos eletrônicos ${ }^{4}$ e a de internet ${ }^{5}$, possíveis distúrbios psiquiátricos contemporâneos, não estarem no DSM-IV, pesquisadores concentraram seus esforços para essas psicopatologias serem apresentadas no DSM-V ${ }^{6,7}$. Este trabalho conjunto gerou a inserção da Internet Gaming Disorder, que é a utilização, de forma patológica, de jogos eletrônicos na internet (o manual psiquiátrico salientou que esse fenômeno necessita de mais estudos para consolidação como transtorno mental) 8 .

Comumente, observa-se o emprego dos termos "uso excessivo" e "dependência" de jogos eletrônicos para designar a mesma condição psiquiátrica, porém essas nomenclaturas não detêm o mesmo significado ${ }^{9}$. Sugere-se que a dependência de jogos eletrônicos não seja restrita apenas ao uso excessivo. Os sintomas atualmente considerados para essa dependência são: saliência, tolerância, modificação do humor, retrocesso, recaída, conflito e problemas na área social ${ }^{10}$.

Pesquisas epidemiológicas revelam variações significativas, com predominância em sujeitos do sexo masculino. Um estudo de extensão nacional recrutou 902 entrevistados, com idades entre 14-81 anos. Os resultados mostraram que a prática de jogos eletrônicos, em geral, é uma atividade difundida e popular entre a população holandesa. A prevalência de jogo problemático na amostra total é de 1,3\%. Entre os adolescentes e adultos jovens, essa prevalência ocorre em 3,3\% dos casos. Particularmente, adolescentes do sexo masculino parecem ser mais vulneráveis a desenvolver hábitos problemáticos na prática de jogos eletrônicos ${ }^{11}$.

Em Singapura, uma pesquisa longitudinal de dois anos, com 3.034 crianças, sugeriu que 9\% desses sujeitos podem ser dependentes ${ }^{12}$. Na Noruega, uma amostra de 816 sujeitos foi investigada, e os resultados demonstram que $0,6 \%$ da população pode ser dependente e 4,1\% apresentam alguns sintomas ${ }^{13}$.

Um estudo de neuroimagem ${ }^{14}$ em usuários de jogos eletrônicos, especificamente sobre as diferenças nos gêneros, reforça os resultados epidemiológicos: sujeitos do sexo masculino, submetidos à ressonância magnética funcional (fMRI), apresentaram maior ativação e conectividade funcional no sistema mesocorticolímbico. Os dados indicam um estado motivacional mais elevado dos homens na prática dos jogos eletrônicos, assim como maiores tendências culturais nessa prática, possibilitando um índice superior da incidência dessa dependência nesse sexo.

No tocante à etiologia, considera-se que indivíduos impulsivos, com baixa autoestima e/ou dificuldades de relacionar-se com terceiros, possuam maior predisposição" ${ }^{11}$ A literatura científica sugere que a dependência de jogos eletrônicos seja estudada por um viés multidimensional, abrangendo os seguintes tópicos: anamnese em relação às questões familiares, culturais, neurobiológicas, de vulnerabilidade mental e comportamentos incorretos aprendidos ao longo da vida. Ainda de acordo com os autores, outros aspectos de risco incluem alguns traços de personalidade (principalmente neuroticismo e hostilidade), as motivações do usuário para jogar e as características estruturais dos jogos eletrônicos (trabalho em equipe, partida solo, on-line ou off-line) ${ }^{15}$. Pesquisadores ainda sugerem que a história de vida do sujeito, a busca por uma catarse de conteúdo violento, escapismo (fuga das responsabilidades cotidianas pela prática de jogos eletrônicos), socialização com pessoas no mundo virtual e busca de autonomia no universo virtual são fatores de risco para essa dependência ${ }^{16}$. 
Outra modalidade que pode propiciar importantes dados são os exames de neuroimagem ${ }^{17}$, que têm se tornado importantes instrumentos para a compreensão da arquitetura e do funcionamento cerebral normal e patológico ${ }^{18}$. Contudo, a ausência de revisões a respeito da variedade de aspectos metodológicos e as possíveis discrepâncias ou similaridades entre os estudos realizados até agora tornam difícil articular uma compreensão abrangente dos mecanismos cerebrais associados às expressões patológicas e não patológicas do uso de jogos eletrônicos. Portanto, o objetivo do presente artigo foi realizar uma revisão sistemática de manuscritos que utilizaram a neuroimagem no estudo da dependência de jogos eletrônicos a fim de identificar as principais regiões cerebrais alteradas.

\section{MÉTODOS}

Foram realizadas buscas nos seguintes bancos de dados: ScieELO, BVS, Lilacs, Science Direct On Line e PubMed. Não houve data mínima para a pesquisa, sendo considerados os artigos encontrados até julho de 2013. Os descritores utilizados para a presente revisão sistemática da literatura foram: "PET", "SPECT", "MRI", "DTI", "EEG", "imaging", "neuroimaging", "spectroscopy", "functional magnetic ressonance", "structural magnetic ressonance", "tractography", "voxel" e "brain", individualmente cruzados com os descritores "gaming" e "video game addiction". Na ausência de descritores específicos (Medical Subject Headings - MeSH), optou-se por utilizar termos próximos aos tópicos de interesse na pesquisa. Nas bases de dados em português, foram utilizados os mesmos termos, com seus equivalentes na língua portuguesa. Na busca manual, não foram encontradas pesquisas completas e originais em livros-texto.

Os critérios de inclusão foram: a) artigos completos e originais relacionando a neuroimagem à dependência de jogos eletrônicos; b) em inglês, espanhol, alemão, francês ou português. Como ainda não há uma determinação transparente na literatura científica, optamos por inserir estudos sobre "uso problemático", "uso excessivo" e/ou "dependência", também em virtude da escassez de manuscritos sobre essa psicopatologia com a neuroimagem. Os critérios de exclusão foram: a) artigos sem resumo; b) estudos de outras psicopatologias por exames de neuroimagem; c) uso de jogos eletrônicos como modelo de tratamento ou aprendizagens; d) uso da neuroimagem apenas com usuários saudáveis; e) assuntos dissonantes ao campo da psiquiatria; f) a relação exclusiva dos neurotransmissores e jogos eletrônicos; g) artigos sobre a dependência de jogos eletrônicos sem o uso de neuroimagem; $h$ ) tratamentos psicoterapêuticos e/ou farmacológicos para a dependência de jogos eletrônicos.

O fluxograma, que mostra de forma detalhada esse processo, segue o modelo do Preferred Reporting Items for Systematic Reviews and Meta-Analyses (PRISMA) ${ }^{19}$ (Figura 1).

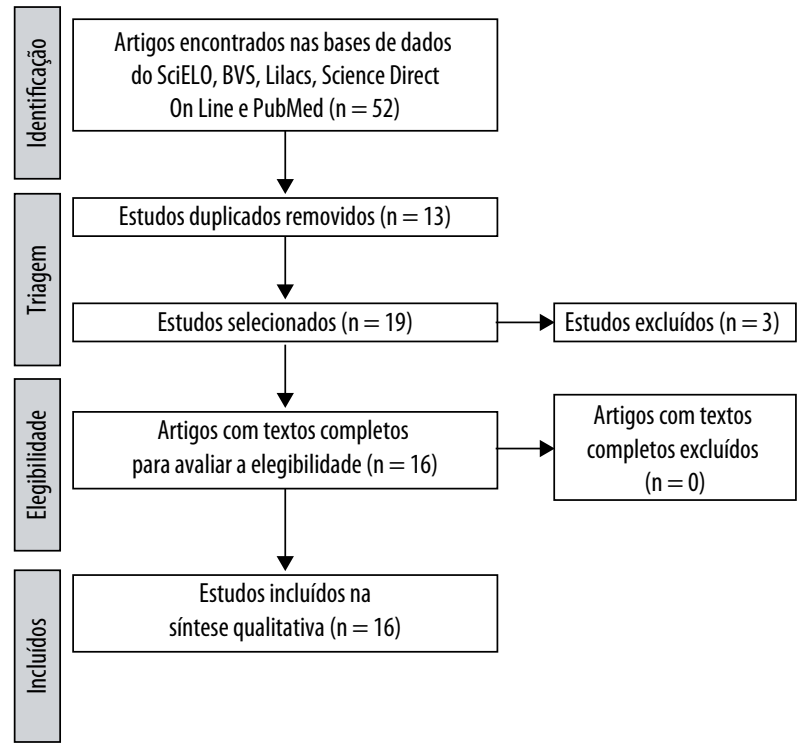

Figura 1. Fluxograma e critérios de seleção e inclusão dos artigos.

\section{RESULTADOS}

A busca encontrou um total de 52 artigos, dos quais 16 foram aproveitados. Com o objetivo de facilitar a visualização dos artigos utilizados, acrescentamos uma tabela com a relação do ano de publicação do artigo e o banco de dados em que o manuscrito foi encontrado (Tabela 1). Foi possível identificar, dessa forma, a existência de um interesse em ascensão pela temática. Nos últimos sete anos, houve crescimento de aproximadamente $87 \%$ no número de artigos publicados. Os 16 artigos utilizados foram divididos de acordo com o tipo de técnica de imageamento: nove de fMRI, quatro de ressonância magnética estrutural (SMRI), um de tomografia por emissão de pósitrons (PET) e dois de eletroencefalograma (EEG).

Tabela 1. Ano de publicação dos artigos e sua frequência nos bancos de dados

\begin{tabular}{lccccccc}
\hline $\begin{array}{l}\text { Anode } \\
\text { publicaçäo } \\
\text { do artigo }\end{array}$ & SciEL0 & BVS & Lilacs & $\begin{array}{c}\text { Science } \\
\text { Direct } \\
\text { On line }\end{array}$ & PubMed & \multicolumn{2}{c}{$\begin{array}{c}\text { Total (duplicados } \\
\text { removidos) }\end{array}$} \\
\hline 2013 & 0 & 0 & 0 & 4 & 4 & 4 & n \\
2012 & 0 & 0 & 0 & 7 & 7 & 7 & $43,75 \%$ \\
2011 & 0 & 0 & 0 & 1 & 1 & 1 & $6,25 \%$ \\
2010 & 0 & 0 & 0 & 0 & 2 & 2 & $12,5 \%$ \\
2009 & 0 & 0 & 0 & 0 & 1 & 1 & $6,25 \%$ \\
2008 & 0 & 0 & 0 & 0 & 0 & 0 & $0 \%$ \\
2007 & 0 & 0 & 0 & 1 & 1 & 1 & $6,25 \%$ \\
Total & 0 & 0 & 0 & 13 & 16 & 16 & $(100.00)$ \\
\hline
\end{tabular}




\section{Ressonância magnética}

Evidencia-se que a técnica de imageamento mais utilizada foi a tomografia por ressonância magnética (RM), estando presente em $81 \%$ dos artigos analisados.

\section{Ressonância magnética funcional}

Utilizando essa técnica, foram selecionados nove artigos de fMRI (Tabela 2).

Tabela 2. Ressonância magnética funcional (fMRI)

\begin{tabular}{|c|c|c|c|c|c|c|c|c|c|}
\hline $\begin{array}{l}\text { Autores } \\
\text { (ano) }\end{array}$ & $\begin{array}{l}\text { Sujeitos } \\
\text { (n) }\end{array}$ & Grupos-P:H/M & $\begin{array}{l}\text { Idade } \\
\text { (desvio-padrão) }\end{array}$ & $\begin{array}{l}\text { Critérios de } \\
\text { inclusão }\end{array}$ & $\begin{array}{l}\text { Critérios de } \\
\text { exclusão }\end{array}$ & $\begin{array}{l}\text { Comorbidade(s)/ } \\
\text { medicamento(s) }\end{array}$ & Resultados & $\begin{array}{l}\text { Tarefa/ } \\
\text { paradigma }\end{array}$ & Contraste \\
\hline $\begin{array}{l}\text { Kühn et al. } \\
(2011)^{20}\end{array}$ & 154 & $\begin{array}{l}\text { a) Usuários com } \\
\text { uso excessivo } \\
\text { (76:52/24) } \\
\text { b) Controle } \\
\text { (78: } 20 / 58)\end{array}$ & $\begin{array}{l}14,4 \pm 0,32 \\
\text { (amostra geral) }\end{array}$ & $\begin{array}{l}\text { 1) Computer } \\
\text { Gaming Behavior } \\
\text { (CSV-S) }\end{array}$ & $\begin{array}{l}\text { Condições } \\
\text { médicas } \\
\text { como tumor, } \\
\text { desordens } \\
\text { neurológicas, } \\
\text { epilepsia } \\
\text { ou outras } \\
\text { psicopatologias }\end{array}$ & Não/Não & $\begin{array}{l}\uparrow \text { de substância cinzenta } \\
\text { e atividade no estriado } \\
\text { ventral (à esquerda) }\end{array}$ & $\begin{array}{l}\text { Monetary } \\
\text { Incentive } \\
\text { Delay (MID) } \\
\text { e Cambridge } \\
\text { Gambling Task } \\
\text { (CGT) }\end{array}$ & $\begin{array}{l}\text { Contraste } \\
\text { comparando } \\
\text { feedback de } \\
\text { qualquer } \\
\text { tipo de perda } \\
\text { (pequena ou } \\
\text { grande) com } \\
\text { feedback sem } \\
\text { perda }\end{array}$ \\
\hline $\begin{array}{l}\text { Han et al. } \\
(2012)^{21}\end{array}$ & 30 & $\begin{array}{l}\text { a) Usuários com } \\
\text { dependência } \\
(15: 15 / 0) \\
\text { b) Controle } \\
\text { (15: } 15 / 0)\end{array}$ & $\begin{array}{l}\text { a) } 14,2 \pm 1,5 \\
\text { (usuários com } \\
\text { dependência) } \\
\text { b) } 14,0 \pm 1,3 \\
\text { (usuários com } \\
\text { uso adaptativo) }\end{array}$ & $\begin{array}{l}\text { 1) Utilizar jogos } \\
\text { eletrônicos } \\
\text { mais de } 4 \text { horas } \\
\text { por dia, e } 30 \\
\text { horas durante a } \\
\text { semana (grupo } \\
\text { de dependentes) } \\
\text { 2) Young Internet } \\
\text { Addiction } \\
\text { Scale (YIAS) > } \\
50 \text { (grupo de } \\
\text { dependentes) } \\
\text { 3) Presença } \\
\text { de conflitos } \\
\text { familiares }\end{array}$ & $\begin{array}{l}\text { Condições } \\
\text { médicas } \\
\text { como tumor, } \\
\text { desordens } \\
\text { neurológicas, } \\
\text { epilepsia, } \\
\text { claustrofobia } \\
\text { e/ou implantes } \\
\text { metálicos }\end{array}$ & Não/Não & $\begin{array}{l}\uparrow \text { do estímulo cerebral no } \\
\text { lobo da ínsula (à direita) } \\
\text { e no giro frontal médio (à } \\
\text { esquerda) }\end{array}$ & $\begin{array}{l}\text { Paradigma de } \\
\text { rastreamento } \\
\text { visual guiado } \\
\text { (sem a } \\
\text { resposta do } \\
\text { participante) }\end{array}$ & $\begin{array}{l}\text { Contraste } \\
\text { comparando } \\
\text { estímulos de } \\
\text { afeto e de um } \\
\text { jogo eletrônico } \\
\text { específico } \\
\text { com estímulos } \\
\text { neutros }\end{array}$ \\
\hline $\begin{array}{l}\text { Han et al. } \\
(2010)^{22}\end{array}$ & 19 & $\begin{array}{l}\text { a) Usuários com } \\
\text { dependência } \\
(11: 11 / 0) \\
\text { b) Controle } \\
(8: 8 / 0)\end{array}$ & $\begin{array}{l}\text { a) } 21,5 \pm 5,6 \\
\text { (usuários com } \\
\text { dependência) } \\
\text { b) 20,3 } \pm 4,1 \\
\text { (usuários com } \\
\text { uso adaptativo) }\end{array}$ & $\begin{array}{l}\text { 1) Utilizar jogos } \\
\text { eletrônicos } \\
\text { mais de } 4 \text { horas } \\
\text { por dia, e } 30 \\
\text { horas durante a } \\
\text { semana (grupo } \\
\text { de dependentes); } \\
\text { 2) Young Internet } \\
\text { Addiction } \\
\text { Scale (YIAS) > } \\
50 \text { (grupo de } \\
\text { dependentes) }\end{array}$ & $\begin{array}{l}\text { Condições } \\
\text { médicas } \\
\text { como tumor, } \\
\text { desordens } \\
\text { neurológicas, } \\
\text { epilepsia, } \\
\text { claustrofobia } \\
\text { e/ou implantes } \\
\text { metálicos }\end{array}$ & $\begin{array}{l}\text { Não/Sim (a } \\
\text { bupropiona }{ }^{24} \\
\text { foi utilizada } \\
\text { durante } 0 \\
\text { estudo) }\end{array}$ & $\begin{array}{l}\uparrow \text { da ativação no giro } \\
\text { para-hipocampal, } \\
\text { cúneus do lobo occipital } \\
\text { (à esquerda), córtex } \\
\text { pré-frontal dorsolateral (à } \\
\text { esquerda) }\end{array}$ & $\begin{array}{l}\text { Paradigma de } \\
\text { rastreamento } \\
\text { visualmente } \\
\text { guiado (sem } \\
\text { a resposta do } \\
\text { participante) }\end{array}$ & $\begin{array}{l}\text { Contraste } \\
\text { comparando } \\
\text { estímulos } \\
\text { de um jogo } \\
\text { eletrônico } \\
\text { específico } \\
\text { com estímulos } \\
\text { neutros }\end{array}$ \\
\hline $\begin{array}{l}\text { Montag } \\
\text { et al. } \\
(2012)^{24}\end{array}$ & 40 & $\begin{array}{l}\text { a) Usuários com } \\
\text { uso excessivo } \\
(21: 21 / 0) \\
\text { b) Controle } \\
\text { (19: } 19 / 0)\end{array}$ & $\begin{array}{l}23,33 \pm 4,45 \\
\text { (amostra geral) }\end{array}$ & $\begin{array}{l}\text { 1) The Personality } \\
\text { Questionnaires } \\
\text { EPQ-R; } \\
\text { 2) Anger Scale of } \\
\text { the ANPS }\end{array}$ & $\begin{array}{l}\text { Diagnósticos } \\
\text { psiquiátricos ou } \\
\text { neurológicos } \\
\text { e/ou uso de } \\
\text { drogas ilícitas }\end{array}$ & Não/Não & $\begin{array}{l}\uparrow \text { da ativação no córtex } \\
\text { frontal e temporal quando } \\
\text { viam fotos do jogo } \\
\downarrow \text { da ativação no } \\
\text { córtex frontal medial } \\
\text { (à esquerda) enquanto } \\
\text { processavam informações } \\
\text { negativas }\end{array}$ & $\begin{array}{l}\text { Paradigma de } \\
\text { rastreamento } \\
\text { visualmente } \\
\text { guiado (sem } \\
\text { a resposta do } \\
\text { participante) }\end{array}$ & $\begin{array}{l}\text { Contraste } \\
\text { comparando } \\
\text { estímulos de } \\
\text { desprazer, } \\
\text { prazer e de um } \\
\text { jogo eletrônico } \\
\text { específico } \\
\text { com estímulos } \\
\text { neutros }\end{array}$ \\
\hline $\begin{array}{l}\text { Dong } \\
\text { et al. } \\
(2012)^{25}\end{array}$ & 29 & $\begin{array}{l}\text { a) Usuários com } \\
\text { dependência } \\
(15: 15 / 0) \\
\text { b) Controle (14: } \\
\text { 14/0) }\end{array}$ & $\begin{array}{l}\text { a) } 24,2 \pm 3,5 \\
\text { (usuários com } \\
\text { dependência) } \\
\text { b) } 24,6 \pm 3,8 \\
\text { (usuários com } \\
\text { uso adaptativo) }\end{array}$ & $\begin{array}{l}\text { 1) Young Internet } \\
\text { Addiction Scale } \\
\text { (YIAS) } \\
>80 \text { (grupo de } \\
\text { dependentes); } \\
\text { 2) Mini } \\
\text { International } \\
\text { Neuropsychiatric } \\
\text { Interview (MINI) }\end{array}$ & $\begin{array}{l}\text { Uso de } \\
\text { medicamentos, } \\
\text { uso de } \\
\text { substâncias e/ } \\
\text { ou diagnósticos } \\
\text { psiquiátricos }\end{array}$ & Não/Não & $\begin{array}{l}\uparrow \text { da homogeneidade } \\
\text { regional (ReHo) no lóbulo } \\
\text { parietal inferior, no } \\
\text { hemisfério cerebelar (à } \\
\text { esquerda) e no giro frontal } \\
\text { médio (à esquerda) } \\
\downarrow \text { da homogeneidade } \\
\text { regional (ReHo) nas } \\
\text { regiões temporal, occipital } \\
\text { e parietal do cérebro }\end{array}$ & $\begin{array}{l}\text { Estado de } \\
\text { repouso }\end{array}$ & Sem contraste \\
\hline
\end{tabular}


Tabela 2. Ressonância magnética funcional (fMRI)

\begin{tabular}{|c|c|c|c|c|c|c|c|c|c|}
\hline $\begin{array}{l}\text { Autores } \\
\text { (ano) }\end{array}$ & $\begin{array}{l}\text { Sujeitos } \\
\text { (n) }\end{array}$ & Grupos-P:H/M & $\begin{array}{l}\text { Idade (desvio- } \\
\text { padrão) }\end{array}$ & $\begin{array}{l}\text { Critérios de } \\
\text { inclusão }\end{array}$ & $\begin{array}{l}\text { Critérios de } \\
\text { exclusão }\end{array}$ & $\begin{array}{l}\text { Comorbidade(s)/ } \\
\text { medicamento(s) }\end{array}$ & Resultados & $\begin{array}{l}\text { Tarefa/ } \\
\text { paradigma }\end{array}$ & Contraste \\
\hline $\begin{array}{l}\text { Ko et al. } \\
(2009)^{26}\end{array}$ & 20 & $\begin{array}{l}\text { a) Usuários com } \\
\text { dependência } \\
\text { (10: } 10 / 0) \\
\text { b) Controle } \\
\text { (10: } 10 / 0)\end{array}$ & $\begin{array}{l}\text { a) } 22,0 \pm 1,49 \\
\text { (usuários com } \\
\text { dependência) } \\
\text { b) } 22,7 \pm 1,34 \\
\text { (usuários com } \\
\text { uso adaptativo) }\end{array}$ & $\begin{array}{l}\text { 1) Chen Internet } \\
\text { Addiction Scale } \\
\text { (CIAS); } \\
\text { 2) Alcohol } \\
\text { Use Disorder } \\
\text { Identification Test } \\
\text { (AUDIT); } \\
\text { 3) Fagerstrom } \\
\text { Test for Nicotine } \\
\text { Dependence } \\
\text { (FTND); } \\
\text { 4) Utilizar mais } \\
\text { de } 30 \text { horas por } \\
\text { semana o jogo } \\
\text { World of Warcraft } \\
\text { (grupo de } \\
\text { dependentes) }\end{array}$ & $\begin{array}{l}\text { Uso de } \\
\text { substâncias } \\
\text { ilegais, } \\
\text { transtornos } \\
\text { psiquiátricos, } \\
\text { retardo } \\
\text { mental e/ou } \\
\text { intolerância } \\
\text { ao exame de } \\
\text { ressonância } \\
\text { magnética }\end{array}$ & Não/Não & $\begin{array}{l}\uparrow \text { da ativação no córtex } \\
\text { orbitofrontal (à esquerda), } \\
\text { núcleo accumbens (à } \\
\text { direita), córtex cingulado } \\
\text { anterior (bilateralmente), } \\
\text { córtex frontal medial, } \\
\text { córtex pré-frontal } \\
\text { dorsolateral (à direita) e } \\
\text { núcleo caudado (à direita) }\end{array}$ & $\begin{array}{l}\text { Paradigma da } \\
\text { reatividade }\end{array}$ & $\begin{array}{l}\text { Contraste } \\
\text { comparando } \\
\text { estímulos } \\
\text { de um jogo } \\
\text { eletrônico } \\
\text { específico } \\
\text { com estímulos } \\
\text { neutros }\end{array}$ \\
\hline $\begin{array}{l}\text { Lorenz } \\
\text { et al. } \\
(2013)^{27}\end{array}$ & 17 & $\begin{array}{l}\text { a) Usuários com } \\
\text { dependência } \\
(8: 8 / 0) \\
\text { b) Controle } \\
(9: 9 / 0)\end{array}$ & $\begin{array}{l}\text { a) } 25,0 \pm 7,4 \\
\text { (usuários com } \\
\text { dependência) } \\
\text { b) } 24,8 \pm 6,9 \\
\text { (usuários com } \\
\text { uso adaptativo) }\end{array}$ & $\begin{array}{l}\text { 1) World of } \\
\text { Warcraft } \\
\text { Addiction } \\
\text { Inventory; } \\
\text { 2) Questionnaire } \\
\text { on Computer } \\
\text { Gaming Behavior } \\
\text { in Childhood; } \\
\text { 3) Entrevista } \\
\text { Clínica } \\
\text { Estruturada para } \\
\text { o DSM-IV (Eixo I) }\end{array}$ & $\begin{array}{l}\text { Diagnósticos } \\
\text { psiquiátricos ou } \\
\text { neurológicos } \\
\text { e/ou uso de } \\
\text { drogas ilícitas }\end{array}$ & Não/Não & $\begin{array}{l}\uparrow \text { da ativação no córtex } \\
\text { pré-frontal medial } \\
\text { e no giro cingulado } \\
\text { anterior após a breve } \\
\text { apresentação, e giro } \\
\text { lingual após a longa } \\
\text { apresentação. } \\
0 \text { post hoc da análise de } \\
\text { conectividade funcional } \\
\text { para a longa apresentação } \\
\text { verificou } \uparrow \text { da ativação } \\
\text { no giro frontal inferior } \\
\text { (à direita), no córtex } \\
\text { orbitofrontal (à esquerda) } \\
\text { e no estriado ventral }\end{array}$ & $\begin{array}{l}\text { Paradigma da } \\
\text { reatividade e } 0 \\
\text { Dot-Probe Task }\end{array}$ & $\begin{array}{l}\text { Contraste } \\
\text { comparando } \\
\text { estímulos de } \\
\text { afeto e de um } \\
\text { jogo eletrônico } \\
\text { específico } \\
\text { com estímulos } \\
\text { neutros }\end{array}$ \\
\hline $\begin{array}{l}\text { Sun et al. } \\
(2012)^{28}\end{array}$ & 20 & $\begin{array}{l}\text { a) Usuários com } \\
\text { dependência } \\
\text { (10: } 10 / 0) \\
\text { b) Controle } \\
\text { (10: } 10 / 0)\end{array}$ & $\begin{array}{l}\text { a) 20,4 } \pm 1,5 \\
\text { (usuários com } \\
\text { dependência) } \\
\text { b) } 20,3 \pm 0,68 \\
\text { (usuários com } \\
\text { uso adaptativo) }\end{array}$ & $\begin{array}{l}\text { 1) Chen's Chinese } \\
\text { Internet addiction } \\
\text { scale (CIAS) > } \\
64 \text { (grupo de } \\
\text { dependentes); } \\
\text { 2) Tempo } \\
\text { de WoW por } \\
\text { semana > } 30 \\
\text { horas (grupo de } \\
\text { dependentes) }\end{array}$ & $\begin{array}{l}\text { Histórico } \\
\text { psiquiátrico } \\
\text { ou histórico } \\
\text { de abuso } \\
\text { de drogas; } \\
\text { histórico de } \\
\text { trauma na } \\
\text { cabeça }\end{array}$ & Não/Não & $\begin{array}{l}\uparrow \text { da ativação no giro } \\
\text { pré-frontal bilateral } \\
\text { dorsal (giro médio frontal, } \\
\text { giro frontal inferior), } \\
\text { lobo temporal, cerebelo, } \\
\text { lobo parietal inferior } \\
\text { (à direita), cúneo, giro } \\
\text { hipocampal (à esquerda), } \\
\text { giro para-hipocampal, } \\
\text { giro fusiforme e núcleo } \\
\text { caudado }\end{array}$ & $\begin{array}{l}\text { Paradigma da } \\
\text { reatividade }\end{array}$ & $\begin{array}{l}\text { Contraste } \\
\text { comparando } \\
\text { estímulos } \\
\text { de um jogo } \\
\text { eletrônico } \\
\text { específico } \\
\text { com estímulos } \\
\text { neutros }\end{array}$ \\
\hline $\begin{array}{l}\text { Ding et al. } \\
(2013)^{29}\end{array}$ & 41 & $\begin{array}{l}\text { a) Usuários com } \\
\text { dependência } \\
\text { (17: 13/4) } \\
\text { b) Controle } \\
\text { (24: } 16 / 8)\end{array}$ & $\begin{array}{l}\text { a) } 16,94 \pm 2,73 \\
\text { (usuários com } \\
\text { dependência) } \\
\text { b) } 15,87 \pm 2,69 \\
\text { (usuários com } \\
\text { uso adaptativo) }\end{array}$ & $\begin{array}{l}\text { 1) Diagnostic } \\
\text { Questionnaire for } \\
\text { Internet Addiction } \\
\text { (YDQ); } \\
\text { 2) Mini } \\
\text { International } \\
\text { Neuropsychiatric } \\
\text { Interview for } \\
\text { Children and } \\
\text { Adolescents } \\
\text { (MINI-KID) }\end{array}$ & $\begin{array}{l}\text { Histórico de } \\
\text { abuso de } \\
\text { substâncias ou } \\
\text { dependências, } \\
\text { hospitalizações } \\
\text { devidas a } \\
\text { transtornos } \\
\text { psiquiátricos e/ } \\
\text { ou ocorrência } \\
\text { de outras } \\
\text { psicopatologias }\end{array}$ & Não / Não & $\begin{array}{l}\uparrow \text { da conectividade } \\
\text { funcional no lobo } \\
\text { posterior do cerebelo } \\
\text { (bilateralmente) e no giro } \\
\text { temporal medial } \\
\downarrow \text { da conectividade } \\
\text { funcional no lobo parietal } \\
\text { inferior e o giro temporal } \\
\text { inferior (à direita) }\end{array}$ & $\begin{array}{l}\text { Estado de } \\
\text { repouso }\end{array}$ & Sem contraste \\
\hline
\end{tabular}

$\uparrow$ : aumento; $\downarrow$ : diminuição; P: número de participantes; H: homem; M: mulher; \pm : mais ou menos (desvio-padrão).

No primeiro estudo ${ }^{20}$, os pesquisadores realizaram dois tipos de análises, a morfometria baseada em voxel (VBM) e a fMRI. Esse foi o único estudo da presente revisão que utilizou dois tipos de imageamento em uma mesma pesquisa. Foram recrutados 154 adolescentes, com idade média de 14,4 anos ( $D P= \pm 0,32$ ) (os autores não informaram a idade média e o desvio-padrão dos dois grupos, apenas da amostra geral), que foram divididos em dois grupos: usuários com uso excessivo (76 participantes, sendo 52 homens e 24 mulheres) e o grupo controle (78 participantes, sendo 20 homens e 58 mulheres), sem diferenças significativas no nível escolar dos participantes. Todos os participantes realizaram o Monetary 
Incentive Delay (MID) e o Cambridge Gambling Task (CGT). O contraste do estudo comparou o feedback de qualquer tipo de perda (pequena ou grande) com feedback sem perda.

Os resultados apontaram aumento de volume e de atividade na substância do corpo estriado (à esquerda). Esse achado correlacionou-se negativamente com tempo de deliberação e aumento de atividade durante a Monetary Incentive Delay (os participantes respondem, por um botão, a um alvo apresentado de forma rápida para tentar ganhar ou evitar perder dinheiro).

Os dados revelaram que, apesar dos distintos exames utilizados (estrutural e funcional), houve uma interseção em relação às alterações no corpo estriado. Outros aspectos relevantes foram: a) estudo que utilizou a maior população na presente revisão, o que permite uma análise amostral de maior profundidade; b) apresentação de uma população mista (apenas dois manuscritos de fMRI recrutaram ambos os sexos). Acreditamos, como limitação, que os autores poderiam ter explorado os dados comparativos em relação ao sexo masculino e feminino. Foi salientado apenas que, no grupo de dependentes, a maior parte era de homens.

No segundo manuscrito ${ }^{21}, 30$ adolescentes, todos do sexo masculino, com idade média de 14 anos, foram divididos em dois grupos, cada um com 15 participantes: usuários com dependência, com idade média de 14,2 anos (DP $= \pm 1,5)$, e o grupo controle, com idade média de 14 anos $(\mathrm{DP}= \pm 1,3)$, sem diferenças significativas no nível escolar dos participantes. Todos foram submetidos ao paradigma de rastreamento visual guiado (sem a resposta do participante) e o contraste comparou estímulos de afeto e de um jogo eletrônico específico com estímulos neutros.

Os resultados demonstraram aumento da ativação cerebral no lobo da ínsula (à direita) e no giro frontal médio (à esquerda), no grupo de dependentes. Um importante aspecto foi o fato de os adolescentes dependentes de jogos eletrônicos apresentarem, concomitantemente, famílias disfuncionais. Assim, consideramos que os resultados poderiam ser distintos se houvesse um núcleo familiar estável. O modelo terapêutico familiar, utilizado pelos pesquisadores com esses dependentes, diminuiu o estímulo cerebral nas áreas cerebrais mencionadas. Acreditamos que a hipótese dos autores em utilizar adolescentes com famílias disfuncionais foi reforçar esse critério como um dos possíveis modelos etiológicos para a dependência de jogos eletrônicos. Esse foi o único estudo que demonstrou um modelo psicoterapêutico para o grupo de dependentes. Porém, uma importante limitação foi que não demonstraram, de forma detalhada, como ocorreu o tratamento desses sujeitos.

$\mathrm{Na}$ terceira pesquisa ${ }^{22}, 19$ adultos jovens, todos do sexo masculino, foram divididos em dois grupos: usuários com dependência (11 participantes), com idade média de 21,5 anos $(\mathrm{DP}= \pm 5,6)$ (dependentes de Star Craft, jogo pertencente ao gênero estratégia (jogado on-line) e grupo controle (oito par- ticipantes), com idade média de 20,3 anos (DP $= \pm 4,1$ ). Não existiram diferenças no nível escolar dos participantes. Os indivíduos realizaram o paradigma de rastreamento visualmente guiado (sem a resposta do participante), e o contraste comparou estímulos de um jogo eletrônico específico com estímulos neutros. Foi apresentado o uso da bupropiona ${ }^{23}$ (fármaco comumente utilizado no tratamento da dependência de nicotina) com os pacientes dependentes.

As áreas cerebrais que apresentaram maior ativação foram o giro para-hipocampal, o cúneus do lobo occipital (à esquerda) e o córtex pré-frontal dorsolateral (à esquerda). 0 estudo demonstrou que, após seis semanas com o uso do medicamento, houve diminuição na ativação das áreas previamente mencionadas, resultando em melhoria dos aspectos cognitivos e emocionais dos pacientes. Esse foi o único estudo da presente revisão que revelou a utilização de um medicamento com os participantes, demonstrando, inicialmente, resultados satisfatórios, porém ainda não é possível desvendar se haverá manutenção dos ganhos em longo prazo após o término do tratamento. Ainda segundo os autores, o uso do medicamento é justificado por haver relação sintomatológica no estado de fissura dos dependentes de substâncias com os dependentes de jogos eletrônicos.

O próximo manuscrito ${ }^{24}$ restringiu-se aos usuários do jogo Counter Strike, pertencente ao gênero de ação, um título de tiro em primeira pessoa (do inglês first-person shooter). Quarenta participantes, todos do sexo masculino, foram divididos em dois grupos: usuários com uso excessivo (21 participantes) e grupo controle (19 participantes). A idade média das amostras foi de 23,33 anos ( $D P= \pm 4,45$ ), sem diferenças significativas no nível escolar. Os usuários realizaram um paradigma de rastreamento visualmente guiado (sem a resposta do participante), e o contraste comparou estímulos de desprazer, prazer e de um jogo eletrônico específico com estímulos neutros.

Os exames acusaram maior ativação no córtex frontal e temporal e menor ativação no córtex frontal medial (à esquerda), enquanto processavam informações negativas (cenas de desastres e pessoas desfiguradas). De acordo com os autores, os resultados descritos podem sugerir um mecanismo de proteção contra a experiência de emoções negativas pela baixa regulação da atividade no sistema límbico, o que levaria a uma experiência de dessensibilização, prolongando o tempo de jogo.

A quinta pesquisa ${ }^{25}$ recrutou 29 sujeitos, todos do sexo masculino, que foram divididos em dois grupos: usuários com dependência (15 participantes), com idade média de $24,2$ anos ( $D P= \pm 3,5)$, e grupo controle (14 participantes), com idade média de 24,6 anos ( $D P= \pm 3,8$ ), sem diferenças significativas no nível escolar. Os participantes realizaram o estudo em estado de repouso, sem o uso de contraste. Por meio da análise de homogeneidade regional (do inglês, regional homogeneity - ReHo), foram relatados aumento da 
ReHo no lóbulo parietal inferior, no hemisfério cerebelar (à esquerda) e no giro frontal médio (à esquerda), regiões responsáveis pela coordenação sensório-motora, e diminuição da ReHo nas regiões temporal ${ }^{24}$, occipital e parietal do cérebro, regiões responsáveis pela função visual e auditiva.

O estudo seguinte ${ }^{26}$ descreveu o resultado de 20 indivíduos do sexo masculino, que foram separados em dois grupos, com o mesmo número de participantes ( $n=10)$ : usuários com dependência, com idade média de 22 anos ( $D P=$ $\pm 1,49$ ), e grupo controle, com idade média de 22,7 anos (DP $= \pm 1,34)$ sem diferenças significativas no nível escolar. Foi utilizado o paradigma da reatividade com o contraste comparando estímulos de um jogo eletrônico específico com estímulos neutros.

O manuscrito objetivou localizar e compreender as regiões cerebrais relacionadas ao desejo de utilizar o jogo World of Warcraft (WoW) de forma excessiva (título de estratégia). Os resultados apontaram para maior ativação do córtex orbitofrontal (à esquerda), núcleo accumbens (à direita), córtex cingulado anterior (bilateralmente), córtex frontal medial, córtex pré-frontal dorsolateral (à direita) e núcleo caudado (à direita). Houve interseção de uma área cerebral com o estudo realizado com os usuários dependentes de outro jogo do mesmo gênero ${ }^{22}$ : o córtex pré-frontal dorsolateral. Tanto o WoW como o Star Craft possuem uma mecânica que induz o jogador a uma notável necessidade de controle visual (espacial), mnemônica e de estratégias. Acreditamos que a prática consecutiva e prolongada desses jogos (do gênero estratégia), assim como de outros títulos desse gênero (League of Legends e DOTA 2), pode estar relacionada às alterações cerebrais citadas pelos pesquisadores.

Uma pesquisa conduzida na Alemanha ${ }^{27}$ demonstrou novos resultados com dependentes de WoW. Dezessete participantes, todos do sexo masculino, foram divididos em dois grupos: usuários com dependência (oito participantes), com idade média de 25 anos ( $D P= \pm 7,4$ ), e grupo controle (nove participantes), com idade média de 24,8 anos ( DP $= \pm 6,9$ ), sem diferenças significativas no nível escolar.

Os autores sugerem, a priori, a condução de estudos de neuroimagem com usuários de jogos on-line, por sua maior capacidade aditiva, em vez dos jogos off-line. Foram realizados o paradigma da reatividade e o Dot-Probe Task, com o contraste comparando estímulos de afeto e desse jogo eletrônico com estímulos neutros. Os estímulos utilizados para a tarefa de atenção visual foram imagens de WoW e de outros jogos, além de figuras da International Affective Picture System, com valência positiva e neutra. Foi optado por aplicar esse paradigma em dois momentos: apresentação breve (viés de atenção) e apresentação longa (reatividade ao estímulo). Os participantes considerados dependentes apresentaram viés de atenção aos dois estímulos visuais relacionados aos jogos eletrônicos e ao estímulo afetivo com valência positiva. Em contraste, o grupo controle não apresentou qualquer tipo de efeito.
As regiões cerebrais dos usuários dependentes que suscitaram maior resposta na apresentação breve foram: córtex pré-frontal medial ${ }^{26}$ e giro cingulado anterior; para a apresentação longa: giro lingual. Em uma análise exploratória post hoc da análise de conectividade funcional, para a apresentação longa, ela se mostrou maior no giro frontal inferior (à direita), no córtex orbitofrontal (à esquerda) ${ }^{26}$ e no estriado ventral ${ }^{20}$.

Um trabalho ${ }^{28}$ contou com 20 participantes, todos do sexo masculino, que foram divididos em dois grupos com o mesmo número de participantes ( $n=10)$ : usuários com dependência, com idade média de 20,4 anos ( $D P= \pm 1,5$ ) e grupo controle, com idade média de 20,3 anos ( $D P= \pm 0,68$ ). Não existiram diferenças no nível escolar dos participantes. Foi utilizado o paradigma da reatividade, com o contraste comparando estímulos de um jogo eletrônico específico com estímulos neutros.

Houve uma avaliação funcional, cuja estimulação experimental envolveu dois momentos de imagens: figuras do WoW e imagens de outros jogos, com as mesmas cores e iluminação do WoW. No grupo de dependentes houve aumento de atividade nas seguintes áreas: giro pré-frontal bilateral dorsal (giro médio frontal, giro frontal inferior), lobo tempora|24,25, cerebelo, lobo parietal25 inferior (à direita), cúneo, giro hipocampal esquerdo, giro para-hipocampal, giro fusiforme e núcleo caudado ${ }^{26}$. Nenhuma dessas áreas teve alterações no grupo controle. Os autores sugerem que as imagens de WoW induzem os jogadores dependentes a um estado de fissura no uso desse jogo, semelhante ao fenômeno no grupo de uso de substâncias. Desse modo, os pesquisadores reforçam que a prática excessiva de WoW pode levar a um comportamento de dependência, principalmente pela ligação dessas estruturas cerebrais ao sistema dopaminérgico vinculado a recompensas e perdas.

Os resultados do último estudo ${ }^{29}$ avaliaram a conectividade cerebral por meio das imagens de fMRI. Quarenta e um sujeitos foram divididos em dois grupos: usuários com dependência (17 participantes, sendo 13 homens e 4 mulheres), com idade média de 16,94 anos ( $D P= \pm 2,73$ ) e grupo controle (24 participantes, sendo 16 homens e 8 mulheres), com idade média de 15,87 anos ( $D P= \pm 2,69$ ). Não existiram diferenças no nível escolar dos participantes. O estudo foi feito com os participantes em estado de repouso, sem o uso contraste.

Os resultados demonstraram que, comparados ao grupo controle, os sujeitos com dependência exibiram aumento da conectividade funcional no lobo posterior do cerebelo (bilateralmente), resultado semelhante ao do estudo anterior ${ }^{28}$, e no giro temporal medial. O lobo parietal inferior e o giro temporal inferior direito exibiram diminuição da conectividade. O manuscrito não apresentou quais são as diferenças entre o grupo do sexo masculino e feminino, limitação essa também encontrada no primeiro estudo. 
Apesar de os estudos terem utilizado critérios distintos na seleção dos participantes (intensidade dos sintomas, número da amostra e sexo dos participantes), os resultados dos manuscritos demonstraram que é possível observar, entre as pesquisas descritas, achados semelhantes nas alterações de algumas áreas cerebrais.

\section{Ressonância magnética estrutural}

Foram encontrados quatro artigos de sMRI (Tabela 3).

Com o intuito de fazer uma investigação mais global, ou seja, sem identificar regiões de interesse a priori, alguns estudos optaram por analisar estruturalmente o cérebro de pacientes com dependência de jogos eletrônicos utilizando uma técnica conhecida como morfometria baseada em voxel (do inglês voxel-based morphometry) (VBM).

Apenas um estudo na presente revisão realizou uma comparação entre três grupos ${ }^{30}$. Cinquenta e cinco participantes, todos do sexo masculino, foram divididos em três grupos: usuários com dependência (19 participantes), com idade média de 20,9 anos ( $D P= \pm 2,0)$; usuários profissionais
(18 participantes), com idade média de 20,8 anos ( $D P= \pm 1,5$ ); e usuários com uso moderado (18 participantes), com idade média de 20,9 anos ( $D P= \pm 2,0$ ). Não existiram diferenças no nível escolar dos participantes.

Os resultados apontam para aumento de substância cinzenta no tálamo (à esquerda) e diminuição de substância cinzenta no giro temporal inferior, região vinculada ao processamento visual. Os autores mencionam que os achados podem indicar um considerável aumento de impulsividade no grupo de dependentes, tendo em vista a inabilidade do controle desse impulso, o que poderia ocasionar dependência de jogos eletrônicos.

A próxima pesquisa ${ }^{31}$ recrutou 34 participantes, que foram divididos em dois grupos: usuários com dependência (17 participantes, sendo 4 homens e 13 mulheres), com idade média de 16,25 anos ( $D P= \pm 3,02$ ), e grupo controle (17 participantes, sendo 2 homens e 15 mulheres), com idade média de 15,54 anos ( $D P= \pm 3,19$ ), sem diferenças no nível escolar. Os dependentes de jogos eletrônicos apresentaram significativa atrofia na substância cinzenta do córtex orbito-

Tabela 3. Ressonância magnética estrutural (sMRI)

\begin{tabular}{|c|c|c|c|c|c|c|c|}
\hline $\begin{array}{l}\text { Autores } \\
\text { (ano) }\end{array}$ & Sujeitos (n) & Grupos-P:H/M & Idade (desvio-padrão) & Critérios de inclusão & Critérios de exclusão & $\begin{array}{l}\text { Comorbidade(s)/ } \\
\text { medicamento(s) }\end{array}$ & Resultados \\
\hline $\begin{array}{l}\text { Han et al. } \\
(2012)^{30}\end{array}$ & 55 & $\begin{array}{l}\text { a) Usuários com } \\
\text { dependência } \\
\text { (19: 19/0) } \\
\text { b) Usuários } \\
\text { profissionais } \\
\text { (17: 17/0) } \\
\text { c) Usuários com } \\
\text { uso moderado } \\
\text { (18: 18/0) }\end{array}$ & $\begin{array}{l}\text { a) } 20,9 \pm 2,0 \\
\text { (usuários } \\
\text { dependentes) } \\
\text { b) } 20,8 \pm \\
1,5 \text { (usuários } \\
\text { profissionais) } \\
\text { c) } 20,9 \pm 2,1 \\
\text { (usuários com uso } \\
\text { moderado) }\end{array}$ & $\begin{array}{l}\text { 1) Young Internet } \\
\text { Addiction Scale (YIAS) }\end{array}$ & $\begin{array}{l}\text { Inventário Beck de } \\
\text { Depressão (BDI) > } 19 \\
\text { Transtornos psiquiátricos } \\
\text { ou histórico de trauma na } \\
\text { região craniana }\end{array}$ & Não/Não & $\begin{array}{l}\uparrow \text { de substância cinzenta no } \\
\text { tálamo (à esquerda) } \\
\downarrow \text { de substância cinzenta no } \\
\text { giro temporal inferior, giro } \\
\text { occipital médio (à direita) e giro } \\
\text { occipital inferior (à esquerda) } \\
\text { (ambos os resultados do grupo de } \\
\text { dependentes de jogos eletrônicos } \\
\text { em comparação ao grupo de uso } \\
\text { moderado) }\end{array}$ \\
\hline $\begin{array}{l}\text { Weng et al. } \\
(2013)^{31}\end{array}$ & 34 & $\begin{array}{l}\text { a) Usuários com } \\
\text { dependência } \\
\text { (17: 4/13) } \\
\text { b) Controle } \\
\text { (17: } 2 / 15)\end{array}$ & $\begin{array}{l}16,25 \pm 3,02 \\
\text { (usuários com } \\
\text { dependência) } \\
15,54 \pm 3,19 \\
\text { (usuários com uso } \\
\text { adaptativo) }\end{array}$ & $\begin{array}{l}\text { 1) Young Internet } \\
\text { Addiction Scale (YIAS) }\end{array}$ & $\begin{array}{l}\text { Transtornos psiquiátricos } \\
\text { ou histórico de trauma na } \\
\text { região craniana }\end{array}$ & Não/Não & $\begin{array}{l}\downarrow \text { de substância cinzenta do } \\
\text { córtex orbitofrontal, ínsula } \\
\text { bilateral e área motora } \\
\text { suplementar (à direita) } \\
\downarrow \text { de substância branca do lobo } \\
\text { frontal bilateral e na cápsula } \\
\text { externa (à direita) }\end{array}$ \\
\hline $\begin{array}{l}\text { Yuan et al. } \\
(2013)^{32}\end{array}$ & 36 & $\begin{array}{l}\text { a) Usuários com } \\
\text { dependência } \\
\text { (18: } 12 / 6) \\
\text { b) Controle } \\
\text { (18: } 12 / 6)\end{array}$ & $\begin{array}{l}\text { 19,5 } \pm 2,8 \text { (usuários } \\
\text { com dependência) } \\
\text { 19,4 } \pm 3,1 \\
\text { (usuários com uso } \\
\text { adaptativo) }\end{array}$ & $\begin{array}{l}\text { 1) Young Diagnostic } \\
\text { Questionnaire for Internet } \\
\text { addiction (YDQ); } \\
\text { 2) Entrevista Clínica } \\
\text { Estruturada para } 0 \\
\text { DSM-IV }\end{array}$ & $\begin{array}{l}\text { Existência de uma desordem } \\
\text { neurológica avaliada } \\
\text { pelo SCID do DSM-IV; } \\
\text { identificação, por exame } \\
\text { de urina, de abuso de } \\
\text { álcool, nicotina ou outras } \\
\text { drogas, no momento do } \\
\text { escaneamento; gravidez ou } \\
\text { período menstrual; doenças } \\
\text { físicas como tumor cerebral, } \\
\text { hepatite ou epilepsia }\end{array}$ & Não/Não & $\begin{array}{l}\downarrow \text { de espessura cortical no córtex } \\
\text { orbitofrontal, córtex insular, } \\
\text { giro lingual, giro pós-central (à } \\
\text { direita), córtex entorrinal e córtex } \\
\text { parietal inferior } \\
\uparrow \text { de espessura cortical no } \\
\text { córtex pré-central (à esquerda), } \\
\text { pré-cúneos, córtex frontal medial, } \\
\text { córtex temporal inferior e córtex } \\
\text { temporal medial }\end{array}$ \\
\hline $\begin{array}{l}\text { Dong et al. } \\
(2012)^{33}\end{array}$ & 31 & $\begin{array}{l}\text { a) Usuários com } \\
\text { dependência } \\
\text { (16: 16/0) } \\
\text { b) Controle } \\
\text { (15: } 15 / 0)\end{array}$ & $\begin{array}{l}\text { a) } 22,2 \pm 3,3 \\
\text { (usuários com } \\
\text { dependência) } \\
\text { b) } 21,6 \pm 2,6 \\
\text { (usuários com uso } \\
\text { adaptativo) }\end{array}$ & $\begin{array}{l}\text { 1) Young Internet } \\
\text { Addiction Scale (Y/IAS); } \\
\text { 2) Mini International } \\
\text { Neuropsychiatric } \\
\text { Interview (MINI) }\end{array}$ & $\begin{array}{l}\text { Transtornos psiquiátricos } \\
\text { ou histórico de trauma na } \\
\text { região craniana }\end{array}$ & Não/Não & $\begin{array}{l}\uparrow \text { da integridade de substância } \\
\text { branca no tálamo e no córtex } \\
\text { cingulado posterior (à esquerda) }\end{array}$ \\
\hline
\end{tabular}

$\uparrow:$ aumento; $\downarrow$ : diminuição; P: número de participantes; H: homem; M: mulher; \pm : mais ou menos (desvio-padrão). 
frontal, ínsula bilateral, áreas também descritas nos estudos de $f M^{121,26,27}$, e área motora suplementar (à direita). Outros resultados revelaram uma redução no corpo caloso, relacionada com a atenção focada, na substância branca do lobo frontal bilateral e na cápsula externa (à direita). Os autores sugerem que essas anormalidades na microestrutura cerebral de substância cinzenta e branca estão presentes em sujeitos dependentes de jogos eletrônicos, o que poderia explicar o mecanismo neuronal desse grupo de sujeitos.

As divergências nos resultados encontrados nos estudos podem estar relacionadas a alguns fatores intrínsecos das amostras. No primeiro trabalho, a população estudada é maior em número de participantes, os grupos estão uma faixa etária superior e o grupo de dependentes possui mais tempo de uso dos jogos eletrônicos. Porém, é relevante a presença, nesta pesquisa, de mais sujeitos do sexo feminino em relação ao sexo masculino e, apesar dos dados epidemiológicos demonstrarem a predominância dessa dependência em homens, as mulheres apresentaram alterações cerebrais semelhantes. Dessa forma, cogitamos se o maior índice de dependência no sexo masculino também pode ocorrer (não consideramos esse como o único fator, mas como um deles) devido a fatores culturais na prática de jogos eletrônicos.

Um estudo ${ }^{32}$ realizado na China avaliou seus participantes durante oito meses. Trinta e seis participantes foram divididos em dois grupos: usuários com dependência (18 participantes, sendo 12 homens e 6 mulheres), com idade média de 19,5 anos (DP $= \pm 2,8$ ), e grupo controle (18 participantes, sendo 12 homens e 6 mulheres), com idade média de 19,4 anos (DP $= \pm 3,1)$. Não existiram diferenças no nível escolar dos participantes.

Os sujeitos colaboraram na tarefa solicitada pelos pesquisadores: o Stroop Color Word Test. Esse procedimento emprega um desenho de bloco em três condições: congruente, incongruente e descanso. Três palavras (vermelho, azul e verde) foram mostradas como o estímulo congruente e incongruente. Cada participante foi instruído a responder à cor mostrada o mais rápido possível, pressionando um botão com a mão direita. Os resultados do estudo demonstraram que o nível de dependência de jogos eletrônicos on-line foi de $12,1 \%$ na amostra investigada, número esse relativamente maior que a média mundial ${ }^{11-13}$. De acordo com as entrevistas realizadas com os participantes sobre o uso da internet e de jogos eletrônicos, foi revelado que eles gastavam uma média de 10,2 $\pm 2,6$ horas ao dia jogando na internet, resultado superior ao do grupo controle: $0,8 \pm 0,4$ hora ao dia. Além disso, o grupo dependente cometeu mais erros em relação ao grupo controle na condição incongruente $(8,56 \pm 4,77$ vs. $4,56 \pm 2,93 ; p<0,05)$.

Os exames de neuroimagem revelaram que diversas regiões cerebrais dos jogadores dependentes apresentaram espessura significativamente reduzida em relação ao grupo controle: córtex orbitofrontal (-9\%), córtex insular (-10\%), giro lingual (-10\%), giro pós-central (à direita) (-13\%), córtex entorrinal (-13\%) e córtex parietal inferior (-10\%). As regiões que apresentaram aumento de espessura cortical foram: córtex pré-central (à esquerda) (+14\%), pré-cúneo $(+13 \%)$, córtex frontal medial $(+10 \%)$, córtex temporal inferior $(+11 \%)$ e córtex temporal medial $(+11 \%)$.

Outra modalidade de ressonância estrutural é o uso de imagens por tensor de difusão (do inglês, diffusion tensor imaging - DTI). Um estudo levantado com 31 participantes, todos do sexo masculino, que foram divididos em dois grupos, sem diferenças no nível escolar: usuários com dependência (16 participantes), com idade média de 22,2 anos ( $\mathrm{DP}= \pm 3,3)$, e grupo controle (15 participantes), com idade média de 21,6 anos ( $D P= \pm 2,6$ ), identificou que regiões de substância branca próximas ao tálamo e ao córtex cingulado posterior possuem altos valores de anisotropia fracionada $(\mathrm{AF})^{33} \mathrm{em}$ pacientes dependentes de jogos eletrônicos.

\section{Tomografia por emissão de pósitrons}

Apenas um artigo sobre PET na dependência de jogos eletrônicos foi encontrado (Tabela 4). Salientamos a necessidade de mais estudos de PET com esse público para que possamos comparar, com maior amplitude, os resultados encontrados em diferentes pesquisas.

Uma pesquisa foi conduzida com 20 participantes, todos do sexo masculino, divididos em dois grupos: usuários com dependência (11 participantes), com idade média de 23,5 anos ( $D P= \pm 2,9)$, e grupo controle (9 participantes), com idade média de 24,7 anos ( $D P= \pm 2,4)$. Não existiram diferenças no nível escolar dos participantes. Os resultados ${ }^{34}$ apontaram para diferenças significativas nas regiões cerebrais no metabolismo da glicose entre os grupos. Os usuários dependentes apresentaram aumento no metabolismo da glicose no giro orbitofrontal médio (à direita), núcleo caudado (à esquerda), lobo da ínsula direita; e diminuição no metabolismo no giro pós-central (bilateralmente), giro pré-central (à esquerda) e lobo occipital (bilateralmente).

O estudo foi realizado com dependentes de jogos eletrônicos que têm sua prática em jogos on-line. Dessa forma, segundo os pesquisadores, existe uma possibilidade de que os jogadores dependentes de jogos eletrônicos off-line possam apresentar bases neurobiológicas distintas do grupo de dependentes de jogos eletrônicos on-line. Apesar de termos encontrado apenas um estudo de PET, ele apresentou regiões semelhantes às de outros estudos que utilizaram outras modalidades de imageamento (córtex orbitofronta|26,27,32, lobo occipital25,34, ínsula ${ }^{21,31}$ e núcleo caudado2,28).

\section{Eletroencefalograma}

Apenas dois estudos de EEG foram encontrados (Tabela 5). 
Tabela 4. Tomografia de emissão de pósitrons (PET)

\begin{tabular}{|c|c|c|c|c|c|c|c|}
\hline Autores (ano) & Sujeitos (n) & Grupos - P:H/M & Idade (desvio-padrão) & Critérios de inclusão & Critérios de exclusão & $\begin{array}{l}\text { Comorbidade(s)/ } \\
\text { medicamento(s) }\end{array}$ & Resultados \\
\hline $\begin{array}{l}\text { Park et al. } \\
(2010)^{34}\end{array}$ & 20 & $\begin{array}{l}\text { a) Usuários com } \\
\text { dependência } \\
\text { (11: } 11 / 0) \\
\text { b) Controle }(9: 9 / 0)\end{array}$ & $\begin{array}{l}\text { a) } 23,5 \pm 2,9 \\
\text { (usuários com } \\
\text { dependência) } \\
\text { b) } 24,7 \pm 2,4 \\
\text { (usuários com uso } \\
\text { adaptativo) }\end{array}$ & $\begin{array}{l}\text { 1) Korean Internet } \\
\text { Game Addiction Scale } \\
\text { (IGS); } \\
\text { 2) Barret Impulsiveness } \\
\text { Scale Version } 11 \\
\text { (BIS-11) }\end{array}$ & $\begin{array}{l}\text { Usuários com história } \\
\text { de uso ou dependência } \\
\text { de substâncias; } \\
\text { episódios psicóticos; } \\
\text { hospitalização } \\
\text { por transtornos } \\
\text { psiquiátricose } \\
\text { tratamento para } \\
\text { psicopatologias } \\
\text { não relacionadas } \\
\text { às dependências } \\
\text { tecnológicas }\end{array}$ & Não/Não & $\begin{array}{l}\uparrow \text { do metabolismo da glicose no } \\
\text { giro orbitofrontal médio (à direita), } \\
\text { núcleo caudado (à esquerda), lobo } \\
\text { da ínsula (à direita) } \\
\downarrow \text { do metabolismo no giro } \\
\text { pós-central (bilateralmente), giro } \\
\text { pré-central (à esquerda) e lobo } \\
\text { occipital (bilateralmente) }\end{array}$ \\
\hline
\end{tabular}

$\uparrow:$ aumento; $\downarrow$ : diminuição; P: número de participantes; H: homem; M: mulher; \pm : mais ou menos (desvio-padrão).

Tabela 5. Eletroencefalograma (EEG)

\begin{tabular}{|c|c|c|c|c|c|c|c|}
\hline Autores (ano) & Sujeitos (n) & Grupos-P:H/M & Idade (desvio-padrão) & Critérios de inclusão & Critérios de exclusão & $\begin{array}{l}\text { Comorbidade(s)/ } \\
\text { medicamento(s }\end{array}$ & Resultados \\
\hline $\begin{array}{l}\text { Thalemann } \\
\text { et al. }(2007)^{35}\end{array}$ & 30 & $\begin{array}{l}\text { a) Usuários com } \\
\text { dependência }(15: 15 / 0) \\
\text { b) Controle (15: } 15 / 0)\end{array}$ & $\begin{array}{l}\text { a) } 28,75 \pm 6,11 \\
\text { (usuários com } \\
\text { dependência) } \\
\text { b) } 25,73 \pm 8,14 \\
\text { (usuários com uso } \\
\text { adaptativo) }\end{array}$ & $\begin{array}{l}\text { Questionnaire of } \\
\text { Differentiated Assessment } \\
\text { of Addiction }\end{array}$ & $\begin{array}{l}\text { Transtornos } \\
\text { psiquiátricos ou } \\
\text { histórico de trauma na } \\
\text { região craniana }\end{array}$ & Não/Não & $\begin{array}{l}\uparrow \text { atividade no lobo } \\
\text { parietal }\end{array}$ \\
\hline $\begin{array}{l}\text { Littel et al. } \\
(2012)^{36}\end{array}$ & 52 & $\begin{array}{l}\text { a) Usuários com } \\
\text { dependência }(25: 23 / 2) \\
\text { b) Controle (27: 10/17) }\end{array}$ & $\begin{array}{l}\text { a) } 20,52 \pm 2,95 \\
\text { (usuários com } \\
\text { dependência) } \\
\text { b) } 21,42 \pm 2,59 \\
\text { (usuários com uso } \\
\text { adaptativo) }\end{array}$ & $\begin{array}{l}\text { Video Game Addiction } \\
\text { Test (VAT) > } 2.5 \text { (grupo } \\
\text { de dependentes) }\end{array}$ & $\begin{array}{l}\text { Transtornos } \\
\text { psiquiátricos ou } \\
\text { histórico de trauma na } \\
\text { região craniana }\end{array}$ & Não/Não & $\begin{array}{l}\uparrow \text { atividade no lobo } \\
\text { parietal }\end{array}$ \\
\hline
\end{tabular}

१: aumento; $\downarrow$ : diminuição; P: número de participantes; H: homem; M: mulher; \pm : mais ou menos (desvio-padrão).

No primeiro estudo ${ }^{35}$, que contou com 30 participantes, todos do sexo masculino, os sujeitos foram divididos em dois grupos com a mesma quantidade de participantes ( $n=15)$, sem diferenças no nível escolar: usuários com dependência, com idade média de 28,75 anos (DP $= \pm 6,11$ ), e grupo controle, com idade média de 25,73 anos (DP $= \pm 8,14)$. Os indivíduos foram separadamente expostos a um estímulo visual padrão de jogos eletrônicos (Doom 3, Counter Strike, Warcraft III) e em seguida expostos a imagens relacionadas ao álcool. Os resultados da pesquisa revelaram que não houve diferenças significativas entre os dois grupos em relação ao uso de cigarro por dia: $M=5,6(D P=8,74)$, no grupo de jogadores dependentes; e $M=2,8$ (DP $=6,14)$, no grupo controle. Porém, quando comparado o estado de fissura entre os dois grupos, os dados demonstraram uma diferença significativa: grupo de jogadores dependentes $(M=73,33$; $D P=16,76)$ e grupo controle $(M=28,89 ; \mathrm{DP}=21,47)$.

De acordo com os autores, a única diferença eletrofisiológica entre os grupos foi nos eletrodos parietais, que exibiram maior resposta quando as imagens de jogos eletrônicos eram vistas pelo grupo de jogadores dependentes. As imagens, segundos os pesquisadores, foram percebidas com maior impacto emocional por esse grupo.

O segundo manuscrito ${ }^{36}$ teve como objetivo investigar o processamento de erro e inibição de resposta em jogadores dependentes de jogos eletrônicos. Cinquenta e dois participantes foram divididos em dois grupos: usuários com dependência (25 participantes, sendo 23 homens e duas muIheres), com idade média de 20,52 anos (DP $= \pm 2,95$ ) e grupo controle (27 participantes, sendo 10 homens e 17 mulheres), com idade média de 21,42 anos (DP $= \pm 2,59$ ). Não existiram diferenças no nível escolar dos participantes.

Os sujeitos foram apresentados ao paradigma Go/NoGo. Os resultados demonstraram: a) os jogadores dependentes apresentaram maior nível de impulsividade $(M=7,24$; DP = $5,00)$ em relação ao grupo controle $(M=4,85 ; D P=3,16) ; b)$ erraram mais na resposta $\mathrm{NoGo}(54 \%, \mathrm{M}=40,12 ; \mathrm{DP}=11,93)$ em relação ao grupo controle (41\%, $M=30,67$; DP $=12,17 ; \mathrm{P}$ $<0,01)$. Os resultados indicaram que jogadores dependentes exibiram maior impulsividade em relação ao grupo controle. Além disso, mostraram diminuição na sensibilidade e maior resposta no paradigma Go/NoGo. Segundo os pesquisado- 
res, esse dado é o mesmo em diversos estudos com populações impulsivas, uso de substâncias e jogo patológico. Assim, como a pesquisa anterior ${ }^{35}$, a região parietal apresentou semelhante alteração nos resultados.

\section{DISCUSSÃO}

O uso de técnicas de neuroimagem tem sido de grande importância no estudo das diversas doenças que acometem o sistema nervoso, pois permitem observar, in vivo, alterações funcionais e estruturais ${ }^{37,38}$. Nos anos 1990, a tomografia por ressonância magnética foi eleita a técnica-padrão para estudo do sistema nervoso central graças à sua alta sensibilidade e, por isso, tem sido utilizada com maior frequência nos estudos das áreas de Neurologia e Psiquiatria ${ }^{37-39}$.

O enorme potencial de informação intrínseco às diferentes sequências de imagem de RM levou a um crescente desenvolvimento de diferentes metodologias quantitativas ${ }^{40}$. Isso possibilitou uma melhora na especificidade dos achados e aplicações de métodos de análise mais confiáveis cientificamente, resultando em avaliações estruturais e funcionais do cérebro ${ }^{37,38}$.

Os resultados encontrados nos estudos de neuroimagem auxiliam na compreensão da dependência de jogos eletrônicos como uma possibilidade psicopatológica, especialmente no que se refere às regiões cerebrais vinculadas ao estado de fissura $22,26,28,41$, à dificuldade de controle dos impulsos ${ }^{36} \mathrm{e}$ às alterações dopaminérgicas ${ }^{28}$, sintomas esses verificados também em dependentes de substâncias.

Em relação às alterações funcionais e estruturais, elas foram mais observadas no lobo frontal (ênfase: córtex préfrontal dorsolateral, córtex orbitofrontal, giro pré-frontal, giro frontal médio), parietal, temporal (ênfase: giro para-hipocampal), núcleos da base, tálamo, ínsula e cerebelo. As técnicas mais utilizadas foram as de RM. Apenas um artigo de PET foi utilizado e não foram achados artigos de SPECT ou espectroscopia.

Consideramos que ainda não há um substrato neuroanatômico para a dependência de jogos eletrônicos, assim como não há modelos etiológicos aprofundados nessa temática. Apesar dessas lacunas, os exames de neuroimagem apresentaram interessantes achados. O primeiro deles é em relação ao lobo frontal. Consideramos que essa região foi constantemente mencionada no presente artigo (giro frontal médio, giro frontal inferior direito, córtex pré-frontal dorsolateral, córtex frontal, córtex frontal medial, córtex orbitofrontal, córtex pré-frontal dorsolateral, córtex pré-frontal medial).

Assim como em dependentes de jogos eletrônicos, alterações nessa região também estão vinculadas à dependência de substâncias ${ }^{42-45}$, facilitando a busca do usuário em relação à droga. Os autores dos estudos de neuroimagem ${ }^{20-36}$ apontaram a associação dos seus resultados com o grupo dependente de substâncias, especialmente no que se refere ao estado de fissura. Sabe-se que uma das funções do lobo frontal seria a de impedir pensamentos negativos, distrações e sentimentos. É possível inferir, por exemplo, que usuários de jogos eletrônicos ou de drogas, que apresentem disfunção no córtex frontal medial, possam ter maior dificuldade em resistir às emoções e aos pensamentos que o induzam a repetir a experiência de dependência ${ }^{46-48}$.

Foi salientado que se confrontar frequentemente com cenas de violência (comuns em jogos de tiro em primeira pessoa) pode criar uma habituação aos efeitos de estímulos considerados aversivos, o que também poderia resultar numa menor ativação frontal ${ }^{24}$. Os autores sugerem que, em relação à dependência, há um mecanismo de defesa do cérebro que, por meio da dessensibilização, potencializa a prática contínua de jogos eletrônicos de forma patológica. Acreditamos que essa explicação possui limitações, tendo em vista que o jogo em questão (Counter Strike) $)^{24}$ é um dos menos violentos no gênero. $O$ potencial aditivo, acreditamos, é maior devido à simplicidade mecânica do jogo e de sua vasta disseminação e aceitação no mercado de jogos eletrônicos. Além disso, por ser um jogo utilizado, quase sempre, on-line, não há finitude, o que prolongaria o tempo de prática. Um estudo de neuroimagem (fMRI), conduzido com população de jogadores saudáveis, demonstrou que a violência presente nesses jogos não interfere, de forma impactante, na prática contínua de jogos eletrônicos ${ }^{49}$.

Especificamente em relação ao córtex orbitofrontal $\left.\right|^{26,31,32,34}$, os pesquisadores sugerem que esses dados são similares às regiões ativadas no estado de fissura de usuários de substâncias (essa região, relacionada à tomada de decisões, é comumente descrita em estudos de neuroimagem com dependentes de maconha ${ }^{50}$, cocaína ${ }^{51}$ e metanfetamina ${ }^{52}$, enfatizando a hipótese dos autores das pesquisas de neuroimagem com dependentes de jogos eletrônicos). De acordo com um dos estudos ${ }^{27}$, essa região cerebral está vinculada a um alto valor motivacional na busca do estímulo vinculado à dependência. Acreditamos que a interseção sintomatológica observada entre esses dois grupos psicopatológicos foi o estado de fissura e a preocupação em estar diante do objeto de dependência. Sugere-se que esses resultados possam auxiliar na estratégia terapêutica empregada, seja ela a psicoterapia e/ou a farmacologia, por meio de uma busca da inibição cognitiva dessa região cerebral27.

Além do que foi exposto, alguns estudos ainda mencionam que o córtex orbitofronta|26,27,32 desempenha importante papel nas conexões biológicas associadas à aprendizagem e à recompensa, guiando as decisões do usuário. Comumente, alterações nessa região estão vinculadas à maior dificuldade no controle dos impulsos e na tomada de decisão $0^{53,54}$. Em usuários dependentes de jogos eletrônicos, é possível que exista uma associação não linear entre o metabolismo da glicose na região orbitofrontal e a impulsividade ${ }^{34}$. Então, 
a atividade metabólica, seja ela muito elevada ou muito baixa, resulta em controle do impulso anormal. Uma pesquisa realizada por PET com dependentes de cocaína também vinculou alterações desse tipo ${ }^{55}$.

A região insular ${ }^{22,33,39}$, também relacionada à tomada de decisões e por aspectos emocionais, pode estar envolvida em ações destrutivas e com a inabilidade de interromper o comportamento aditivo ${ }^{56-58}$. Estudos futuros devem aprofundar a discussão sobre o efeito de disfunções nessa região cerebral em indivíduos dependentes de jogos eletrônicos.

Alterações no pré-cúneo ${ }^{28,32}$ estão relacionadas ao estado de fissura do usuário, tomando como ponto de partida as funções dessa região cerebral: memória, imaginação e atenção ${ }^{59}$. Em dependentes de substâncias, o estado de fissura também está relacionado a alterações em outras regiões descritas nos estudos anteriores, como o córtex temporal inferior e medial ${ }^{60,61}$.

O aumento da espessura cortical no córtex pré-central e no córtex temporal medial, regiões vinculadas ao planejamento e execução dos movimentos ${ }^{62}$, pode ser explicado, possivelmente, pelo uso contínuo e prolongado de periféricos, como mouse, joysticks e teclados, porém isso ainda tem sido pouco discutido na literatura.

O lobo parietal é uma interface relacionada à atenção e à integração de múltiplos sinais sensoriais, que podem ser usadas no planejamento motor ${ }^{63}$. Em relação a essa região, os resultados sugerem um aumento da sincronização na coordenação sensório-motora, assim como uma diminuição da excitabilidade visual e auditiva. Esse aspecto é apontado como uma indução a um condicionamento operante, que resultaria em uma prática frequente de jogos eletrônicos, podendo tornar-se patológica ${ }^{25}$. Os achados ainda demonstraram que, em concordância com os achados com dependentes de substâncias, o uso patológico de jogos eletrônicos é mantido pela dessensibilização (do sistema mesolímbico dopaminérgico) junto à saliência (um componente de recompensa emocional que induz o jogador a necessitar desse estímulo) 9,20,35. Alterações no lobo parietal também são reveladas em estudos de neuroimagem com dependentes de internet ${ }^{64}$ e de nicotina ${ }^{65}$.

O lobo temporal representa uma importante ligação com as funções de memória declarativa e explícita. É uma região cerebral também relacionada à aprendizagem. Diversos estudos mencionaram alterações em regiões do lobo temporal24,25,28-30,32, enfatizando, especialmente, experiências de dessensibilização emocional à prática de jogos eletrônicos de conteúdo violento, corroborando um uso contínuo. Além disso, sugere-se um mecanismo de proteção contra a experiência de emoções negativas pela baixa regulação da atividade no sistema límbico ${ }^{24}$. Observam-se alterações semelhantes em estudos conduzidos com usuários crônicos de maconha ${ }^{66}$ e dependentes de internet ${ }^{67}$ e de álcool ${ }^{68}$.
O núcleo caudado $26,28,34$ é responsável pela convergência das funções cognitivas ${ }^{69}$, assim como memória e aprendizagem. Essa região cerebral é descrita como desempenhando importante papel no controle de comportamentos impulsivos. Sua disfunção pode estar relacionada a uma dificuldade do jogador em interromper o seu comportamento aditivo. Essa informação também é descrita em estudo de neuroimagem com jogadores patológicos (jogos de azar) ${ }^{70}$, dependentes de internet ${ }^{71}$ e usuários de cocaína ${ }^{72}$.

O corpo estriado está relacionado aos receptores de dopamina, assim como no planejamento e modulação das vias de movimento, além de estar potencialmente envolvido em processos cognitivos, como funções executivas e memória de trabalho. Segundo os autores dos estudos ${ }^{20,27}$, alterações nessa região indicam um processamento de recompensa alterado. Também são encontradas alterações dessa natureza em estudos de neuroimagem com dependentes de cocaí$n a^{73}$ e dependentes de internet ${ }^{74,75}$.

O tálamo ${ }^{30,33}$ possui conexões vindas de diversas regiões do cérebro, incluindo regiões corticais, que, em outros estudos, apresentaram aumento do volume de substância cinzenta. Dessa forma, esse achado poderia ser explicado também pela plasticidade cortical ${ }^{76}$. Um estudo de DTI, realizado com dependentes de cocaína, revelou também alterações no tálamo, região vinculada ao estado de abstinência dos usuários ${ }^{77}$. Ainda foi possível verificar que os achados no tálamo se correlacionam à gravidade da dependência ${ }^{33}$. 0 mesmo estudo demonstrou que essas alterações podem ser sinais de uma vulnerabilidade preexistente, ao desenvolvimento de dependências, assim como alterações no processamento de recompensas, o que poderia levar o jogador a utilizar os jogos eletrônicos por tempo maior do que pretendido com o intuito de obter satisfação nessa prática, gerando, assim, um possível adoecimento psíquico.

As funções do cerebelo ${ }^{28,29}$ não são limitadas ao controle do movimento, pois ele também desempenha importante papel em processos emocionais e cognitivos. A região posterior do cerebelo está predominantemente envolvida na regulação cognitiva, processamento de sinais e armazenamento de memórias verbal e auditiva. Os estudos ${ }^{28,29}$ que enfatizaram essa região do encéfalo sugerem que adolescentes dependentes de jogos eletrônicos on-line exibem diferentes padrões, em estado de repouso, da atividade encefálica. Essas alterações, segundo os pesquisadores, são parcialmente consistentes com pacientes dependentes de substâncias, ocorrendo, então, uma anormalidade neurobiológica similar a outros transtornos aditivos.

O presente manuscrito apresentou limitações: a) não foram encontradas informações em fontes alternativas; b) o número de artigos encontrados $(n=16)$ ainda é pequeno, porém acreditamos que é um número significativo, havendo crescente interesse pela temática; c) o uso de diferentes métodos de seleção de participantes em cada estudo dificulta 
uma análise mais acurada, especialmente de DTI, PET e EEG; d) a quantidade de participantes variou em cada manuscrito; e) a maioria dos estudos utilizou apenas pacientes do sexo masculino; f) a maioria das pesquisas demonstrou resultados em adolescentes e adultos jovens, desconhecendo-se resultados em crianças e sujeitos mais velhos; g) não há resultados de estudos realizados no Brasil sobre o tema, apenas com populações estrangeiras.

\section{CONCLUSÃO}

A despeito dos métodos utilizados (fMRI, sRMI, PET e EEG), os estudos apontaram convergências quanto às reciprocidades cerebrais. Em relação às alterações funcionais e estruturais, elas foram mais observadas no lobo frontal (ênfase: córtex pré-frontal dorsolateral, córtex orbitofrontal, giro pré-frontal, giro frontal médio), parietal, temporal (ênfase: giro para-hipocampal), núcleos da base, tálamo, ínsula e cerebelo. Essas regiões cerebrais são semelhantes às observadas em pacientes dependentes de substâncias e de internet, especialmente durante o estado de fissura. Apesar de apenas recentemente pesquisas de neuroimagem em dependentes de jogos eletrônicos terem sido realizadas, contamos no momento com achados significativos alinhados à compreensão dos mecanismos neurais associados à dependência de jogos eletrônicos e respectiva inserção como categoria nosológica no âmbito psiquiátrico. Consideramos, por fim, que, apesar de ainda não existir um substrato neuroanatômico para a dependência de jogos eletrônicos, o interesse pela temática é crescente, auxiliando na compreensão e funcionamento dessa possível psicopatologia contemporânea.

\section{CONTRIBUIÇÕES INDIVIDUAIS}

Igor Lins Lemos - Contribuiu na concepção e no desenho do estudo, na análise e interpretação dos dados, elaboração e revisão do artigo e aprovou a versão final a ser publicada.

Paula Rejane Beserra Diniz - Contribuiu na análise e interpretação dos dados, na revisão do artigo e aprovou a versão final a ser publicada.

Julio Fernando Prieto Peres - Contribuiu na análise e interpretação dos dados, na revisão do artigo e aprovou a versão final a ser publicada.

Everton Botelho Sougey - Contribuiu na análise e interpretação dos dados, na revisão do artigo e aprovou a versão final a ser publicada.

\section{CONFLITO DE INTERESSE E SUPORTE FINANCEIRO}

Não houve conflito de interesse ou financiamento para a presente revisão.

\section{REFERÊNCIAS}

1. Peres J, Dias ACS, Suassuna AMV, Almeida MA, Guedes SA, Sanches GP. Cultura tecnológica e vulnerabilidade ao trauma psíquico. 0 mundo da Saúde. 2012;36(2):303-10.

2. Jäger S, Müller KW, Ruckes C, Wittig T, Batra A, Musalek M, et al. Effects of a manualized short-term treatment of internet and computer game addiction (STICA): study protocol for a randomized controlled trial. Trials. 2012;13(43):1-8.

3. Desai RA, Krishnan-Sarin S, Cavallo D, Potenza MN. Video-gaming among high school students: health correlates, gender differences, and problematic gaming. Pediatrics. 2010;126(6):1414-24.

4. Lemos IL, Santana SM. Dependência de jogos eletrônicos: a possibilidade de um novo diagnóstico psiquiátrico. Rev Psiq Clín. 2012;39(1):28-33.

5. Conti MA, Jardim AP, Hearst N, Cordás TA, Tavares H, Abreu CN. Avaliação da equivalência semântica e consistência interna de uma versão em português do Internet Addiction Test (IAT). Rev Psiq Clín. 2012;39(3):106-10.

6. Rooij AJ, Schoenmakers TM, Eijnden RJ, Vermulst AA, Mheen DV. Video Game Addiction Test: validity and psychometric characteristics. Cyberpsychol Behav Soc Netw. 2012;15(9):507-11.

7. Walther B, Morgenstern M, Hanewinkel R. Co-occurrence of addictive behaviours: personality factors related to substance use, gambling and computer gaming. Eur Addict Res. 2012;18(4):167-74.

8. APA. DSM-V: Internet Gaming Disorder [Internet]. 2013. [Capturado em: 01 mai 2013]. Disponivel em: <http://www.dsm5.org/Documents/Internet\%20Gaming\%20Disorder\%20 Fact\%20Sheet.pdf>.

9. Weinstein AM. Computer and video game addiction - A comparison between game users and non-game users. Am J Drug Alcohol Abuse. 2010;36(5):268-76.

10. Lemmens JS, Valkenburg PM, Peter J. Development and validation of a game addiction scale for adolescents. Media Psychology. 2009;12:77-95.

11. Haagsma MC, Pieterse ME, Peters 0 . The prevalence of problematic video gamers in the Netherlands. Cyberpsychol Behav Soc Netw. 2012;15(3):162-8.

12. Gentile DA, Choo H, Liau A, Sim T, Li D, Fung D, et al. Pathological video game use among youths: a two-year longitudinal study. Pediatrics. 2011;127(2):319-29.

13. Mentzoni RA, Brunborg GS, Molde H, Myrseth H, Skouverøe KJ, Hetland J, et al. Problematic video game use: estimated prevalence and associations with mental and physical health. Cyberpsychol Behav Soc Netw. 2011;14(10):591-6.

14. Hoeft F, Watson CL, Kesler SR, Bettinger KE, Reiss AL. Gender differences in the mesocorticolimbic system during computer game-play. J Psychiatr Res. 2008;42(4):253-8.

15. Zboralski K, Orzechowska A, Talarowska M, Darmosz A, Janiak A, Janiak M, et al. The prevalence of computer and internet addiction among pupils. Postepy Hig Med Dosw. 2009;2(63):8-12

16. Shaw M, Black DW. Internet addiction: definition, assessment, epidemiology and clinical management. CNS Drugs. 2008;22(5):353-65.

17. Linares IM, Trzesniak C, Chagas MH, Hallak JE, Nardi AE, Crippa JA. Neuroimaging in specific phobia disorder: a systematic review of the literature. Rev Bras Psiquiatr. 2012;34(1):101-11.

18. Baldaçara L, Filho GMA, Jackowski A. Neuroanatomia funcional e comportamental. In: Kapczinski F, Quevedo J, Izquierdo (orgs.). Bases biológicas dos transtornos psiquiátricos: uma abordagem translacional. Porto Alegre: Artmed; 2011. p. 23-34.

19. Moher D, Liberati A, Tetzlaff J, Altman DG; PRISMA Group. Preferred reporting items for systematic reviews and meta-analyses: the PRISMA statement. J Clin Epidemiol. 2009;62(10):1006-12.

20. Kühn S, Romanowski A, Schilling C, Lorenz R, Mörsen C, Seiferth N, et al. The neural basis of video gaming. Transl Psychiatry. 2011;1(53):1-5.

21. Han DH, Kim SM, Lee YS, Renshaw PF. The effect of family therapy on the changes in the severity of on-line game play and brain activity in adolescents with on-line game addiction. Psychiatry Res. 2012;202(2):126-31.

22. Han DH, Hwang JW, Renshaw PF. Bupropion sustained release treatment decreases craving for video games and cue-induced brain activity in patients with Internet video game addiction. Exp Clin Psychopharmacol. 2010;18(4):297-304.

23. Menossi HS, Goudriaan AE, De Azevedo-Marques Périco C, Nicastri S, de Andrade AG, D'Elia $G$, et al. Neural bases of pharmacological treatment of nicotine dependence - insights from functional brain imaging: a systematic review. CNS Drugs. 2013;27(11):921-41. 
24. Montag C, Weber B, Trautner P, Newport B, Markett S, Walter NT, et al. Does excessive play of violent first-person-shooter-video-games dampen brain activity in response to emotional stimuli? Biol Psychol. 2012;89(1):107-11.

25. Dong G, Huang J, Du X. Alterations in regional homogeneity of resting-state brain activity in internet gaming addicts. Behav Brain Funct. 2012;8(1):41.

26. Ko CH, Liu GC, Hsiao S, Yen JY, Yang MJ, Lin WC, et al. Brain activities associated with gaming urge of online gaming addiction. J Psychiatr Res. 2009;43(7):739-47.

27. Lorenz RC, Krüger JK, Neumann B, Schott BH, Kaufmann C, Heinz A, et al. Cue reactivity and its inhibition in pathological computer game players. Addict Biol. 2013;18(1):134-46.

28. Sun Y, Ying H, Seetohul RM, Xuemei W, Ya Z, Qian L, et al. Brain fMRI study of crave induced by cue pictures in online game addicts (male adolescents). Behav Brain Res. 2012;233(2):563-76.

29. Ding WN, Sun JH, Sun YW, Zhou Y, Li L, Xu JR, et al. Altered default network restingstate functional connectivity in adolescents with Internet gaming addiction. PLOS One. 2013;8(3).

30. Han DH, Lyoo IK, Renshaw PF. Differential regional gray matter volumes in patients with on-line game addiction and professional gamers. J Psychiatr Res. 2012;46(4):507-15.

31. Weng CB, Qian RB, Fu XM, Lin B, Han XP, Niu CS, et al. Gray matter and white matter abnormalities in online game addiction. Eur J Radiol. 2013;82(8):1308-12.

32. Yuan K, Cheng P, Dong T, Bi Y, Xing L, Yu D, et al. Cortical thickness abnormalities in late adolescence with online gaming addiction. PLoS One. 2013;8(1).

33. Dong G, Devito E, Huang J, Du X. Diffusion tensor imaging reveals thalamus and posterior cingulate cortex abnormalities in internet gaming addicts. J Psychiatr Res. 2012;46(9):1212-6.

34. Park HS, Kim SH, Bang SA, Yoon EJ, Cho SS, Kim SE. Altered regional cerebral glucose metabolism in internet game overusers: a 18F-fluorodeoxyglucose positron emission tomography study. CNS Spectr. 2010;15(3):159-66.

35. Thalemann R, Wölfling K, Grüsser SM. Specific cue reactivity on computer game-related cues in excessive gamers. Behav Neurosci. 2007;121(3):614-8.

36. Littel M, van den Berg I, Luijten M, van Rooij AJ, Keemink L, Franken IH. Error processing and response inhibition in excessive computer game players: an event-related potential study. Addict Biol. 2012;17(5):934-47.

37. Tofts $P$ (ed.). Quantitative MRI of the brain: measuring changes caused by disease. Chichester, UK: John Wiley \& Sons; 2004.

38. Griffa A, Baumann PS, Thiran JP, Hagmann P. Structural connectomics in brain diseases. Neuroimage. 2013;15(80):515-26.

39. Klöppel S, Abdulkadir A, Jack CR Jr, Koutsouleris N, Mourão-Miranda J, Vemuri P. Diagnostic neuroimaging across diseases. Neuroimage. 2012;61(2):457-63.

40. Bandettini PA. Twenty years of functional MRI: the science and the stories. Neuroimage. 2012;62(2):575-88.

41. Han DH, Kim YS, Lee YS, Min KJ, Renshaw PF. Changes in cue-induced, prefrontal cortex activity with video-game play. Cyberpsychol Behav Soc Netw. 2010;13(6):655-61.

42. Lu H, Chefer S, Kurup PK, Guillem K, Vaupel DB, Ross TJ, et al. fMRI response in the medial prefrontal cortex predicts cocaine but not sucrose self-administration history. Neuroimage. 2012;62(3):1857-66.

43. Chen BT, Yau HJ, Hatch C, Kusumoto-Yoshida I, Cho SL, Hopf FW, et al. Rescuing cocaineinduced prefrontal cortex hypoactivity prevents compulsive cocaine seeking. Nature. 2013;496(7445):359-62.

44. Goldstein RZ, Tomasi D, Alia-Klein N, Zhang L, Telang F, Volkow ND. The effect of practice on a sustained attention task in cocaine abusers. Neuroimage. 2007:35(1):194-206.

45. Herting MM, Fair D, Nagel BJ. Altered fronto-cerebellar connectivity in alcohol-naïve youth with a family history of alcoholism. Neuroimage. 2011;54(4):2582-9.

46. Ghasemzadeh MB, Vasudevan P, Giles C, Purgianto A, Seubert C, Mantsch JR. Glutamatergic plasticity in medial prefrontal cortex and ventral tegmental area following extendedaccess cocaine self-administration. Brain Res. 2011;219(1413):60-71.

47. Caffino L, Cassina C, Giannotti G, Orrù A, Moro F, Di Clemente A, et al. Short-term abstinence from cocaine self-administration, but not passive cocaine infusion, elevates aCaMKII autophosphorylation in the rat nucleus accumbens and medial prefrontal cortex. Int J Neuropsychopharmacol. 2013;19:1-7.
48. Moreno-López L, Catena A, Fernández-Serrano MJ, Delgado-Rico E, Stamatakis EA, PérezGarcía M, et al. Trait impulsivity and prefrontal gray matter reductions in cocaine dependent individuals. Drug Alcohol Depend. 2012;125(3):208-14.

49. Regenbogen C, Herrmann M, Fehr T. The neural processing of voluntary completed, real and virtual violent and nonviolent computer game scenarios displaying predefined actions in gamers and nongamers. Soc Neurosci. 2010;5(2):221-40.

50. Cousijn J, Wiers RW, Ridderinkhof KR, Van Den Brink W, Veltman DJ, Goudriaan AE. Grey matter alterations associated with cannabis use: results of a VBM study in heavy cannabis users and healthy controls. Neuroimage. 2012;59(4):3845-51.

51. Smith DG, Jones PS, Williams GB, Bullmore ET, Robbins TW, Ersche KD. Overlapping decline in orbitofrontal gray matter volume related to cocaine use and body mass index. Addict Biol. 2013 Aug 8. doi: 10.1111/adb.12081. [Epub ahead of print].

52. Volkow ND, Chang L, Wang GJ, Fowler JS, Ding YS, Sedler M, et al. Low level of brain dopamine D2 receptors in methamphetamine abusers: association with metabolism in the orbitofrontal cortex. Am J Psychiatry. 2001;158(12):2015-21.

53. Schoenbaum G, Roesch M, Stalnaker TA. Orbitofrontal cortex, decision-making and drug addiction. Trends Neurosci. 2006;29(2):116-24.

54. Wallis JD. Orbitofrontal cortex and its contribution to decision-making. Ann Rev Neurosci. 2007:30:31-56

55. Goldstein RZ, Alia-Klein N, Leskovjan AC, Fowler JS, Wang GJ, Gur RC, et al. Anger and depression in cocaine addiction: association with the orbitofrontal cortex. Psychiatry Res. 2005;138(1):13-22

56. Naqvi NH, Bechara A. The hidden island of addiction: the insula. Trends Neurosci. 2009;32(1):56-67.

57. Naqvi NH, Rudrauf D, Damasio H, Bechara A. Damage to the insula disrupts addiction to cigarette smoking. Science. 2007;315(5811):531-4.

58. Naqvi NH, Bechara A. The insula and drug addiction: an interoceptive view of pleasure, urges, and decision-making. Brain Struct Funct. 2010;214(5-6):435-50

59. Wilcox CE, Teshiba TM, Merideth F, Ling J, Mayer AR. Enhanced cue reactivity and frontostriatal functional connectivity in cocaine use disorders. Drug Alcohol Depend. 2011;115(12):137-44.

60. Goudriaan AE, de Ruiter MB, Van Den Brink W, Oosterlaan J, Veltman DJ. Brain activation patterns associated with cue reactivity and craving in abstinent problem gamblers, heavy smokers and healthy controls: an fMRI study. Addict Biol. 2010;15(4):491-503.

61. Gozzi A, Tessari M, Dacome L, Agosta F, Lepore S, Lanzoni A, et al. Neuroimaging evidence of altered fronto-cortical and striatal function after prolonged cocaine self-administration in the rat. Neuropsychopharmacology. 2011;36(12):2431-40.

62. Machado ABM. Neuroanatomia funcional. Rio de Janeiro: Atheneu; 2005

63. Ptak R, Müri RM. The parietal cortex and saccade planning: lessons from human lesion studies. Front Hum Neurosci. 2013;7(7):254.

64. Du W, Liu J, Gao X, Li L, Li W, Li X, et al. Functional magnetic resonance imaging of brain of college students with internet addiction. Zhong Nan Da Xue Xue Bao Yi Xue Ban. 2011:36(8):744-9.

65. Yalachkov Y, Naumer MJ. Involvement of action-related brain regions in nicotine addiction. J Neurophysiol. 2011;106(1):1-3.

66. Batalla A, Bhattacharyya S, Yücel M, Fusar-Poli P, Crippa JA, Nogué S, et al. Structural and functional imaging studies in chronic cannabis users: a systematic review of adolescent and adult findings. PLoS One. 2013;8(2):e55821.

67. Liu J, Gao XP, Osunde I, Li X, Zhou SK, Zheng HR, et al. Increased regional homogeneity in internet addiction disorder: a resting state functional magnetic resonance imaging study. Chin Med J (Engl). 2010;123(14):1904-8.

68. Fein G, Greenstein D, Cardenas VA, Cuzen NL, Fouche JP, Ferrett H, et al. Cortical and subcortical volumes in adolescents with al cohol dependence but without substance or psychiatric comorbidities. Psychiatry Res. 2013;214(1):1-8.

69. Macfarlane MD, Looi JC, Walterfang M, Spulber G, Velakoulis D, Crisby M, et al; the LADIS Study Group. Executive dysfunction correlates with caudate nucleus atrophy in patients with white matter changes on MRI: A subset of LADIS. Psychiatry Res. 2013 Aug 2. doi:10.1016/j.pscychresns.2013.05.010. 
70. Shao R, Read J, Behrens TE, Rogers RD. Shifts in reinforcement signalling while playing slotmachines as a function of prior experience and impulsivity. Transl Psychiatry. 2013;3:e213.

71. Dong G, Hu Y, Lin X, Lu Q. What makes internet addicts continue playing online even when faced by severe negative consequences? Possible explanations from an fMRI study. Biol Psychol. 2013;94(2):282-9.

72. Moeller SJ, Tomasi D, Honorio J, Volkow ND, Goldstein RZ. Dopaminergic involvement during mental fatigue in health and cocaine addiction. Transl Psychiatry. 2012;2:176.

73. Bustamante JC, Barrós-Loscertales A, Costumero V, Fuentes-Claramonte P, Rosell-Negre $P$, Ventura-Campos $N$, et al. Abstinence duration modulates striatal functioning during monetary reward processing in cocaine patients. Addict Biol. 2013 Feb 28. doi: 10.1111/ adb.12041. [Epub ahead of print].
74. Hou H, Jia S, Hu S, Fan R, Sun W, Sun T, et al. Reduced striatal dopamine transporters in people with internet addiction disorder. J Biomed Biotechnol. 2012, Article ID 854524, 5 pages doi:10.1155/2012/854524

75. Kim SH, Baik SH, Park CS, Kim SJ, Choi SW, Kim SE. Reduced striatal dopamine D2 receptors in people with Internet addiction. Neuroreport. 2011;22(8):407-11.

76. Fields RD. Myelination: an overlooked mechanism of synaptic plasticity? Neuroscientist. 2005;11(6):528-31.

77. Bell RP, Foxe JJ, Nierenberg J, Hoptman MJ, Garavan H. Assessing white matter integrity as a function of abstinence duration in former cocaine-dependent individuals. Drug Alcohol Depend. 2011;114(2-3):159-68. 